\title{
Organ of Origin
}

National Cancer Institute

\section{Source}

National Cancer Institute. Organ of Origin. NCI Thesaurus. Code C156840.

The organ identified as the original site of the tumor. 